\title{
INEQUALITIES ON THE RICCI CURVATURE
}

\author{
ADELA MiHAI
}

Abstract. We improve Chen-Ricci inequalities for a Lagrangian submanifold $M^{n}$ of dimension $n(n \geqslant 2)$ in a $2 n$-dimensional complex space form $\widetilde{M}^{2 n}(4 c)$ of constant holomorphic sectional curvature $4 c$ with a semi-symmetric metric connection and a Legendrian submanifold $M^{n}$ in a Sasakian space form $\widetilde{M}^{2 n+1}(c)$ of constant $\varphi$-sectional curvature $c$ with a semi-symmetric metric connection, respectively.

Mathematics subject classification (2010): 53C40, 53B05, 53B15.

Keywords and phrases: Semi-symmetric metric connection, improved Chen-Ricci inequality, complex space form, Sasakian space form, Ricci curvature.

\section{REFERENCES}

[1] K. Arslan, R. Ezentaş, I. Mihai, C. Murathan, C. Ozgur, Certain inequalities for submanifolds in $(k, \mu)$-contact space forms, Bull. Aust. Math. Soc. 64 (2001), 201-212.

[2] K. Arslan, R. Ezentaş, I. Mihai, C. Murathan, C. Ozgur, Ricci curvature of submanifolds in locally conformal almost cosymplectic manifolds, Math. J. Toyama Univ. 26 (2003), 13-24.

[3] B. Y. CHEN, Some pinching and classification theorems for minimal submanifolds, Arch. Math. (Basel) 60 (1993), 568-578.

[4] B. Y. CHEN, Strings of Riemannian invariants, inequalities, ideal immersions and their applications, The Third Pacific Rim Geometry Conference (Seoul, 1996), 7-60, Monogr. Geom. Topology, 25, Int. Press, Cambridge, MA, 1998.

[5] B. Y. CHEN, Relations between Ricci curvature and shape operator for submanifolds with arbitrary codimensions, Glasg. Math. J. 41 (1999), 33-41.

[6] B. Y. CHEN, On Ricci curvature of isotropic and Lagrangian submanifolds in complex space forms, Arch. Math. (Basel) 74 (2000), 154-160.

[7] B. Y. CHEN, Some new obstructions to minimal and Lagrangian isometric immersions, Japan J. Math. 26 (2000), 1-17.

[8] B. Y. CHEn, Pseudo-Riemannian Geometry, $\delta$-Invariants and Applications, World Scientific Publishing Co. Pte. Ltd., Hackensack, NJ, 2011.

[9] S. DENG, An improved Chen-Ricci inequality, Int. Electron. J. Geom. 2 (2009), 39-45.

[10] S. DENG, Improved Chen-Ricci inequality for Lagrangian submanifolds in quaternion space forms, Int. Electron. J. Geom. 5 (2012), 163-170.

[11] H. A. Hayden, Subspaces of a space with torsion, Proc. London Math. Soc. 34 (1932), 27-50.

[12] F. Malek And M. B. K. BALGeshir, Slant submanifolds of almost contact metric 3-structure manifolds, Mediterr. J. Math. 10 (2013), 1023-1033.

[13] K. Matsumoto, I. Minai, A. Oiaga, Ricci curvature of submanifolds in complex space forms, Rev. Roumaine Math. Pures Appl. 46 (2001), no. 6, 775-782.

[14] A. Minai, Modern Topics in Submanifold Theory, Editura Universitatii Bucuresti, Bucharest, 2006.

[15] A. Minai, C. OzGuR, Chen inequalities for submanifolds of real space forms with a semi-symmetric metric connection, Taiwanese J. Math. 14 (2010), 1465-1477.

[16] A. Minai, C. OzGur, Chen inequalities for submanifolds of complex space forms and Sasakian space forms endowed with semi-symmetric metric connections, Rocky Mountain J. Math. 41 (2011), no. 5, 1653-1673.

[17] A. Mihai, I. N. RAdulescu, An improved Chen-Ricci inequality for Kaehlerian slant submanifolds in complex space forms, Taiwanese J. Math. 16 (2012), 761-770. 
[18] I. MinaI, Ricci curvature of submanifolds in Sasakian space forms, J. Aust. Math. Soc. 72 (2002), $247-256$.

[19] I. Mihai, I. N. RAdulescu, An improved Chen-Ricci inequality for Legendrian submanifolds in Sasakian space forms, J. Adv. Math. Stud. 4 (2011), 51-58.

[20] Z. NAKAO, Submanifolds of a Riemannian manifold with semisymmetric metric connections, Proc. Amer. Math. Soc. 54 (1976), 261-266.

[21] A. Oiaga, I. Minai, B. Y. Chen inequalities for slant submanifolds in complex space forms, Demonstratio Math. 32 (1999), 835-846.

[22] C. Ozgur, B. Y. Chen inequalities for submanifolds of a Riemannian manifold of quasi-constant curvature, Turkish J. Math. 35 (2011), 501-509.

[23] C. Ozgur, C. Murathan, Chen inequalities for submanifolds of a locally conformal almost cosymplectic manifold with a semi-symmetric metric connection, An. St. Univ. Ovidius Constanta Ser. Mat. 18 (2010), no. 1, 239-253.

[24] C. Ozgur, C. Murathan, Chen inequalities for submanifolds of a cosymplectic space form with a semi-symmetric metric connection, An. St. Univ. Al. I. Cuza Iasi. Mat. (N.S.) 58 (2012), no. 2, $395-408$.

[25] C. OzGur, A. DE, Chen inequalities for submanifolds of a Riemannian manifold of nearly quasiconstant curvature, Publ. Math. Debrecen 82 (2013), no. 2, 439-450.

[26] C. OzGur, A. Minai, Chen inequalities for submanifolds of real space forms with a semi-symmetric non-metric connection, Canadian Math. Bull. 55 (2012), 611-622.

[27] S. Sular, C. OzGUR, Warped products with a semi-symmetric metric connection, Taiwanese J. Math. 15 (2011), no. 4, 1701-1719.

[28] S. Sular, C. Ozgur, Generalized Sasakian space forms with semi-symmetric metric connections, An. Stiin. Univ. Al. I. Cuza Iasi Mat. (N.S.), 60, 1 (2014), 145-156.

[29] M. M. TRIPATHI, Improved Chen-Ricci inequality for curvature-like tensors and its applications, Differential Geom. Appl. 29 (2011), 685-698.

[30] K. Yano, On semi-symmetric metric connection, Rev. Roumaine Math. Pures Appl. 15 (1970), 1579 1586.

[31] K. Yano, M. Kon, Anti-Invariant Submanifolds, M. Dekker, 1976. 